\title{
ISOMORPHIC POLYNOMIAL RINGS
}

\author{
D. B. COLEMAN AND E. E. ENOCHS
}

ABstract. A ring is called invariant if whenever $B$ is a ring such that the polynomial rings $A[X]$ and $B[X]$ are isomorphic, then $A$ and $B$ are isomorphic. $A$ is strongly invariant if an isomorphism $A[X] \rightarrow B[X]$ maps $X$ onto a $B$-generator of $B[X]$. Strongly invariant rings are invariant. Among the strongly invariants are left perfect rings, local domains and rings of algebraic integers.

1. Introduction. In this paper we consider the following question. Can there be nonisomorphic rings $A$ and $B$ whose polynomial rings $A[X]$ and $B[X]$ are isomorphic? We know of no such pair of rings.

All rings are assumed to have identity elements. The Jacobson radical of a ring is denoted $J(A)$.

A ring $A$ is said to be invariant if whenever $B$ is a ring such that $A[X]$ and $B[X]$ are isomorphic, then $A$ and $B$ are also isomorphic. A tool used in considering this question is the fact that $X \mapsto b_{0}$ $+b_{1} X+\cdots+b_{n} X^{n}$ induces a $B$-algebra surjection if and only if $b_{0}, b_{1}, \cdots, b_{n}$ are all central, $b_{0}$ is a unit, and $b_{1}, \cdots, b_{n}$ are nilpotent, i.e., only under these conditions is $b_{0}+b_{1} X+\cdots+b_{n} X^{n}$ a $B$-generator of $B[X]$. Furthermore any such surjection is an isomorphism.

This had been proved by R. W. Gilmer in the commutative case. Another extension of a well-known commutative theorem that appears here is the following: $A[X]$ has only trivial units if and only if $A$ has no nonzero nilpotent elements. This is used to obtain a sufficient condition on $A$ in order that the description of the units of $A[X]$ coincides with the description in the commutative case.

We say that the ring $A$ is strongly invariant ${ }^{1}$ if whenever $B$ is a ring and $\sigma: A[X] \rightarrow B[X]$ is a ring isomorphism, then $\sigma(X)$ is a $B$ generator. If $A$ is a strongly invariant ring and if $\sigma: A[X] \rightarrow B[X]$ is a ring isomorphism, then $\sigma$ followed by a $B$-algebra isomorphism $B[X] \rightarrow B[X]$ taking $\sigma(X) \mapsto X$ gives an isomorphism $A[X] \rightarrow B[X]$ taking $X \mapsto X$, so that $A \cong A[X] /(X) \cong B[X] /(X) \cong B$. Thus a strongly invariant ring is invariant.

Presented to the Society, April 18, 1970; received by the editors December 15, 1969 and, in revised form, April 15, 1970.

AMS 1969 subject classifications. Primary 1230, 1625.

Key words and phrases. Polynomial ring, group of units, Artinian ring, locally nilpotent ideal, left $T$-nilpotent ideal, Jacobson radical, left perfect ring.

${ }^{1} \mathrm{P}$. Eakin and B. Heinzer have shown that for a field $K, K[X]$ is invariant. It is ohviously not strongly invariant. 
It is shown that if the center of $A$ is a reduced ring (no nilpotent elements) and is generated by its units, then $A$ is a strongly invariant ring. In particular, semisimple Artinian rings are strongly invariant, as are local domains. A class of integral domains that includes algebraic number rings is also shown to be strongly invariant. Using these results it can be deduced that all (left or right) perfect rings are strongly invariant.

2. The image of $X$ under surjections. The following theorem extends Theorem 3 in [3]. As was noted by Gilmer for the commutative case, this theorem is likely to be known by some people, but is probably not in the literature.

THEOREM 1. Let $B$ be a ring and let $b_{0}, b_{1}, \cdots, b_{n}$ be members of $B$ for $n \geqq 1$. Then the following statements are equivalent:

(1) $b_{0}, b_{1}, \cdots, b_{n}$ are central, $b_{1}$ is a unit, and $b_{2}, \cdots, b_{n}$ are nilpotent.

(2) The B-algebra homomorphism of $B[X]$ into $B[X]$ induced by $X \mapsto b_{0}+b_{1} X+\cdots+b_{n} X^{n}$ is a surjection.

(3) The B-algebra homomorphism of $B[X]$ into $B[X]$ induced by $X \mapsto b_{0}+b_{1} X+\cdots+b_{n} X^{n}$ is an isomorphism.

PRoof. That (1) implies (3) follows from the commutative arguments; namely Lemma 1 (b) and the argument on p. 331 preceding Theorem 3 in [3].

We need only prove that (2) implies (1). Let $C$ denote the center of $B$ and suppose that (2) holds. Then since a surjection takes $C[X]$ into $C[X]$, it follows that $b_{0}, b_{1}, \cdots, b_{n}$ are central. Now let $\bar{B}$ $=B /\left(b_{1}\right)$ and $b \in B$, let $\bar{b}=b+\left(b_{1}\right)$. Then $X \mapsto b_{0} X+\cdots+\bar{b}_{n} X^{n}$ induces a surjective $\bar{B}$-homomorphism $\bar{B}[X] \rightarrow \bar{B}[X]$. But $\bar{b}_{1}=0$, so this is impossible unless $\left(b_{1}\right)=B$; i.e., $b_{1}$ is a unit. Let $Y=b_{0}+b_{1} X$ $+\cdots+b_{n} X^{n}$ and $Y^{\prime}=X+b_{1}^{-1} b_{2} X^{2}+\cdots+b_{1}^{-1} b_{n} X^{n}$. Then clearly $B\left[Y^{\prime}\right]=B[Y]=B[X]$; and it is easy to see that if

$$
X=c_{0}+c_{1} Y^{\prime}+c_{2} Y^{\prime 2}+\cdots+c_{m} Y^{\prime m},
$$

$c_{i} \in B$, then each $c_{i} \in C$. Hence by the commutative version, $b_{1}^{-1} b_{i}$ is nilpotent for $i=2, \cdots, n$. Since $b_{1}^{-1}$ is central, this completes the proof.

Corollary 1. Let $A$ and $B$ be rings and suppose that $\sigma: A[X]$ $\rightarrow B[X]$ is a ring surjection such that $\sigma(A) \subset B$. Then $\sigma(A)=B$.

Proof. By hypothesis, $B[\sigma(X)]=B[X]$, so by Theorem 1 there is a $B$-algebra isomorphism $B[X] \rightarrow B[X]$ such that $\tau(\sigma(X))=X$. 
Thus $\tau \sigma: A[X] \rightarrow B[X]$ is a ring surjection with $\tau \sigma(A) \subset B$ and $\tau \sigma(X)=X$. So clearly $\tau \sigma(A)=B$; but $\tau^{-1}(B)=B$, therefore $\sigma(A)=B$.

3. Units in $A[X]$. For a ring $B$, let $B^{*}$ denote the group of units in $B$. First we prove an extension of a well-known theorem about commutative rings.

Theorem 2. Let $A$ be a ring. Then $A[X]^{*}=A^{*}$ if and only if $A$ has no nonzero nilpotent elements.

Proof. If $a$ is a nonzero nilpotent element of $A$, then $1+a X$ is a nonconstant unit in $A[X]$.

Conversely, suppose that $A$ has no nonzero nilpotent elements. Let $f(X)=a_{0}+a_{1} X+\cdots+a_{n} X^{n} \in A[X]$ be a unit and let $g(X)$ $=b_{0}+b_{1} X+\cdots+b_{m} X^{m}$ be its inverse where $n \geqq 1$. Then considering the last coefficient of the product $f(X) g(X)$, we have $a_{n} b_{m}=0$. By induction we show that $a_{n} b_{m-1}=a_{n} b_{m-2}=\cdots=a_{n} b_{0}=0$. Supposing that we have $a_{n} b_{m}=a_{n} b_{m-1}=\cdots=a_{n} b_{m-8}=0$ for $s \geqq 0$, consider the coefficient of $X^{n+m-(\bullet+1)}$ in $f(X) g(X): a_{n} b_{m-(s+1)}+a_{n-1} b_{m-8}$ $+\cdots+a_{n-(s+1)} b_{m}=0$. Since $a_{n} b_{m-k}=0$ for $k=0, \cdots, s, b_{m-k} a_{n}$ is nilpotent, hence zero. Thus multiplying the above equation on the right by $a_{n}$ yields $a_{n} b_{m-(s+1)} a_{n}=0$. Hence $a_{n} b_{m-(s+1)}$ is nilpotent, so is zero. Thus by induction we get $a_{n} b_{0}=0$. But since $a_{0} b_{0}=b_{0} a_{0}=1$, it follows that $a_{n}=0$. Thus the only units in $A[X]$ are the units of $A$.

COROLlaRY 2. If $A$ is a ring without nonzero nilpotent elements, and if $A$ is generated as a ring by $A^{*}$, then $A$ is a strongly invariant ring.

Proof. If $\sigma: A[X] \rightarrow B[X]$ is a ring isomorphism, then clearly $B$ has no nonzero nilpotent elements, so $B[X]^{*}=B^{*}$ and $\sigma\left(A^{*}\right)=B^{*}$. Hence if $A^{*}$ generates $A, \sigma(A)=B$, so clearly $\sigma(X)$ is a $B$-generator of $B[X]$.

COROLlaRy 3. A local ring without nonzero nilpotent elements is strongly invariant.

TheOREM 3. Let $A$ be a ring with center $C$. If $C$ is reduced and is generated by $C^{*}$, then $A$ is strongly invariant.

Proof. Let $\sigma: A[X] \rightarrow B[X]$ be an isomorphism. Then if $D$ denotes the center of $B, \sigma(C[X])=D[X]$. But by Corollary $2 C$ is strongly invariant so $\sigma(X)$ is a $D$-generator of $D[X]$. But then by Theorem 1 it is easy to see that $\sigma(X)$ is a $B$-generator of $B[X]$.

Corollary 4. If $A$ is a ring whose center is a field, then $A$ is a 
strongly invariant ring. In particular, every simple ring is strongly invariant.

COROllaRy 5. Every semisimple Artinian ring is strongly invariant.

Proof. Let $A$ be semisimple Artinian. Then $A$ is the direct sum of a finite number of simple rings. Since the only central idempotents of $A[X]$ are those of $A$, it follows that $A$ is strongly invariant if and only if each of these simple rings is strongly invariant. Each one is strongly invariant by Corollary 4.

It would be interesting to extend Theorem 2 and to give necessary conditions on $A$ in order that $A[X]^{*}$ consists of elements $a_{0}+a_{1} X$ $+\cdots+a_{n} X^{n}$, with $a_{0} \in A^{*}$, and $a_{1}, \cdots, a_{n}$ nilpotent. We conclude this section with some modest progress in this direction.

If $I$ is an ideal in $A$, then the canonical homomorphism $A[X]$ $\rightarrow(A / I)[X]$ and Theorem 2 give

Leмma 1. If $a$ is an ideal in $A$ such that $A / Q$ has no nonzero nilpotent elements, and if $a_{0}+a_{1} X+\cdots+a_{n} X^{n}$ is a unit in $A[X]$, then $a_{0}$ is a unit in $A$ and $a_{1}, a_{2}, \cdots, a_{n}$ are in $a$.

Corollary 6. If the set $\mathfrak{N}$ of nilpotent elements of $A$ forms an ideal in $A$, then a unit in $A[X]$ has the form $a_{0}+a_{1} X+\cdots+a_{n} X^{n}$, with $a_{0} \in A^{*}$ and $a_{1}, \cdots, a_{n} \in \Re$.

Lemma 2. Suppose that $A$ is a ring such that if $x, y \in A$ and $x y=0$, then $y x=0$. Then the set $\Re$ of nilpotent elements of $A$ forms an ideal.

Proof. It suffices to show that if $x^{k}=0$ and if $y_{1}, \cdots, y_{k} \in A$, then $x y_{1} x y_{2} \cdots x y_{k}=0$. Suppose $x^{k}=0$. Then $x^{k} y_{1}=0$, so $x^{k-1} y_{1} x=0$. Thus $x^{k-1} y_{1} x y_{2}=0$, so $x^{k-2} y_{1} x y_{2} x=0$, etc.

TheOREM 4. Suppose that $A$ satifies the hypothesis of Lemma 2. Then $A[X]^{*}$ consists of elements of the form $a_{0}+a_{1} X+\cdots+a_{n} X^{n}$, with $a_{0} \in A^{*}$ and $a_{1}, \cdots, a_{n}$ nilpotent.

Proof. That units take on the desired form follows from Lemma 2 and Corollary 6.

Conversely, suppose $Y=a_{0}+a_{1} X+\cdots+a_{n} X^{n}$ with $a_{0} \in A^{*}$ and $a_{1}, \cdots, a_{n}$ nilpotent. We may assume that $a_{0}=1$. Then the inverse of $Y$ in the formal power series ring $A[[X]]$ is $1+b_{1} X+\cdots+b_{m} X^{m}$ $+\cdots$, where $b_{m}$ is the sum of terms of the form $a_{i_{1}} a_{i_{2}} \cdots a_{i_{k}}$, where $i_{1}+i_{2}+\cdots+i_{k}=m$. It follows from the proof of Lemma 2 that there is a positive integer $N$ such that $b_{m}=0$ for $m \geqq N$. Thus $Y^{-1} \in A[X]$. This completes the proof. 
Let $J(A)$ denote the Jacobson radical of $A$. Amitsur [1] has proved that $J(A[X])=N[X]$, where $N=J(A[X]) \cap A$ and $N$ is a nil ideal in $A$. Thus the following proposition is of some interest. The proof is similar to that of Theorem 4.

Proposition. Suppose that $A$ is a ring whose nilpotent elements form an ideal $\Re$. Then the following statements are equivalent:

(a) $J(A[X])=\Re[X]$.

(b) $A[X]^{*}$ consists of elements of form $a_{0}+a_{1} X+\cdots+a_{n} X^{n}$, where $a_{0} \in A^{*}$ and $a_{1}, a_{2}, \cdots, a_{n}$ are nilpotent.

Note that in general $A[X]^{*}$ and the set of elements of the form $a_{0}+a_{1} X+\cdots+a_{n} X^{n}$ with $a_{0} \in A^{*}$ and $a_{1}, \cdots, a_{n}$ nilpotent are not comparable. For let $A$ be the ring of $2 \times 2$ matrices over the rational numbers. The element

$$
\left(\begin{array}{ll}
1 & 0 \\
0 & 1
\end{array}\right)+\left(\begin{array}{ll}
0 & 0 \\
1 & 0
\end{array}\right) X+\left(\begin{array}{ll}
0 & 1 \\
0 & 0
\end{array}\right) X^{2}
$$

has the desired form but is not a unit. On the other hand, the unit

$$
\left(\begin{array}{rr}
1 & 1 \\
-1 & 1
\end{array}\right)+\left(\begin{array}{ll}
1 & 0 \\
1 & 0
\end{array}\right) X
$$

has no nilpotent coefficient.

4. Strongly invariant domains. Let $D$ be an integral domain with field of fractions $K$, and let $S$ be the set of nonzero elements in the ring generated by $D^{*}$. Let $D^{(1)}=S^{-1} D$, the elements in $K$ of the form $s^{-1} d, d \in D, s \in S$. Define $D^{(n)}$ inductively for $n>1$ by $D^{(n)}$ $=\left(D^{(n-1)}\right)^{(1)}$. Let $\tilde{D}$ denote the union of the ascending chain $D^{(1)}$ $\subset D^{(2)} \subset \cdots$. Notice that $\widetilde{D}^{(1)}=\widetilde{D}$ since a unit in $\tilde{D}$ must be in some $D^{(n)}$, so that its inverse is in $D^{(n+1)} \subset \widetilde{D}$.

THEOREM 5. Let $D$ be an integral domain such that $\tilde{D}=K$. Then $D$ is a strongly invariant ring.

Proof. Let $E$ be a ring such that $D[X] \cong E[X]$. Since units in $E[X]$ are units in $E$ it follows that any isomorphism $D[X] \rightarrow E[X]$ can be extended to an isomorphism $D^{(1)}[X] \rightarrow E^{(1)}[X]$; and any isomorphism $D^{(n)}[X] \rightarrow E^{(n)}[X]$ can be extended to an isomorphism $D^{(n+1)}[X] \rightarrow E^{(n+1)}[X]$. Hence there is an isomorphism $\sigma: \widetilde{D}[X]$ $\rightarrow \tilde{E}[X]$. But $\tilde{D}=K$, a field, so $\sigma(\tilde{D}) \subset \tilde{E}$. But then $\sigma(D) \subset E$, so as before $\sigma(D)=E$.

COROLLARY 7. Let $D$ be the ring of algebraic integers in an algebraic number field $K$. Then $D$ is a strongly invariant ring. 
Proof. $D^{(1)}=K$.

Note that if $L$ is a field and $A$ a subring of $L$ such that $L$ is algebraic over the field of fractions of $A$ then $\widetilde{B}$ is the field of fractions of $B$ for any subring $B$ of $L$ with $A \subset B$. Hence in fact any subring of an algebraic extension of the rational numbers is strongly invariant.

\section{Perfect rings.}

ThEOREM. Let $A$ be a ring and let $N=J(A)$. Suppose that $A$ satisfied the conditions

(i) $J(A[X])=N[X]$, and

(ii) $A / N$ is strongly invariant.

Then $A$ is strongly invariant.

Proof. Let $\sigma: A[X] \rightarrow B[X]$ be a ring isomorphism. From $[1]$ and the invariance of the radical under isomorphisms we have that $\sigma(N[X])=J(B[X])=M[X]$ for a nil ideal $M$ of $B$. Write $\sigma(X)=b_{0}$ $+b_{1} X+\cdots+b_{n} X^{n} ; b_{i} \in B$. Consider the isomorphism $A / N[X]$ $\rightarrow B / M[X]$ given by

$$
(A / N)[X] \rightarrow A[X] / N[X] \rightarrow B[X] / M[X] \rightarrow(B / M)[X],
$$

where the middle isomorphism is induced by $\sigma$ and the others are the obvious ones. Under this isomorphism, $X \mapsto b_{0}+\bar{b}_{1} X$ $+\cdots+\bar{b}_{n} X^{n}$, where $\bar{b}_{i}$ denotes the coset $b_{i}+M$ in $B / M$. Since $A / N$ is strongly invariant, $b_{i}$ is a unit in $B / M$. But $M \subset J(B)$, so $b_{1}$ is a unit in $B$. And $b_{2}, \cdots, b_{n}$ are nilpotent elements of $B / M$, so, since $M$ is nil, $b_{2}, \cdots, b_{n}$ are nilpotent elements of $B$. Thus $\sigma(X)$ is a $B$-generator. This completes the proof.

If $N$ is locally nilpotent-that is, if every finitely generated subring of $N$ is nilpotent, then $N[X]$ is a nil ideal, so hypothesis (i) holds. In particular, if $N$ is left or right $T$-nilpotent [2] the condition holds. A left (right) perfect ring is a ring $A$ such that $N$ is left (right) $T$ nilpotent and $A / N$ is semisimple Artinian [2]. Hence

COROLlARY. A left or right perfect ring is strongly invariant.

Corollary. A left or right Artinian ring is strongly invariant.

\section{REFERENCES}

1. S. A. Amitsur, Radicals of polynomial rings, Canad. J. Math. 8 (1956), 355-361. MR 17, 1179.

2. Hyman Bass, Finitistic dimension and a homological generalization of semiprimary rings, Trans. Amer. Math. Soc. 95 (1960), 466-488. MR 28 \#1212.

3. R. W. Gilmer, Jr., R-automorphisms of $R[X]$, Proc. London Math. Soc. (3) 18 (1968), 328-336. MR 37 \#5207.

University of Kentucky, Lexington, Kentucky 40506 